\title{
Emotion Recognition Using Finger Tip Temperature: First Step towards an Automatic System
}

\author{
G. Shivakumar and P. A. Vijaya
}

\begin{abstract}
FTT) has been used as a sensitive index to monitor if a person is relaxed or not. When a person is relaxed, his/her vessels will be dilated and finger tip will be warmer. If one is anxious or tense, the vessels will be constricted and finger tip will be cool. FTT is a method of bio-feedback to tell the patient if he/she is in a relaxed condition. The subjects experiencing emotion in a higher magnitude differ from those who can regulate the emotions and such a factor is termed as emotional intelligence. In this paper, the subject whose FTT is to be measured is shown a video clipping or photographs or recorded audio signals are played. The subject, upon the influence of these, will respond and emotions are evoked. The change in FTT due to this evoked emotion is recorded for a finite interval of time and then analyzed. In this work, a total of 75 subjects are chosen. During selection of subjects, enough care is taken to include persons from different age groups, gender and social background. The data corresponding to FTT is acquired and processed using National Instrument's LabVIEW software and hardware tools. The results obtained are encouraging with high level of accuracy and repeatability. Since, the subjects also give feedback about the emotion experienced, the obtained results are validated.
\end{abstract}

Index Terms-Emotion, lab view, stimulus, TDM

\section{INTRODUCTION}

Without emotions, life is experienced as having little meaning or purpose, and the pleasures that are derived from rewarding experiences are considerably reduced. Emotionless actions are often related to machines, which execute a sequence of pre-programmed commands. While this common-place perception of the role emotions play in everyday life is pertinent as it is somewhat limited in scope. Our physical body and mind are interactive. Physical disease may cause mental disorders and mental discomfort may have adverse influence on physical health. When a person is subjected to stress beyond what he or she can withstand, physical or mental disorders may therefore develop. The mechanism is that the stress, when our brain senses it, may cause dysfunction of our automatic nervous system, endocrine system and immune system via the effect of neurological pathways.

In clinical practice, biofeedback machine is used to find a way to detect an emotional tension caused by stress. But in the conventional method of measuring finger tip temperature, the biofeedback machine is large and expensive. It has the sensor which contacts the patient's skin to detect the finger tip temperature. The measured temperature value is converted into an electronic signal

Manuscript received May 12, 2012; revised June 23, 2012.

The authors are with the Department of Instrumentation at Malnad College of Engineering, Hassan, India. which is displayed on a monitor to tell the patient if he/she is in a relaxed condition. Since it is impossible that a medical staff or a large machine is always readily available for the patients, it is desirable to develop an auxiliary medical instrument which can be conveniently worn by the people and help the wearer or adjust them at any time.

\section{STIMULUS For EMOTIONS}

Emotional content can modify and update the goals and consequently alter the direction of attention to the presented stimuli. Emotions and goals are strongly intertwined in the sense that the immediate relevance of any stimulus to a goal defines the emotionality of the stimulus. The relationship between emotions and the personal goals and concerns of individuals is often suggested to be the basis for emotion elicitation and differentiation by appraisal theorists. For instance the emotional tag of fear can be attached to a threatening stimulus in so far as the latter can potentially impede the goal of survival. Another example is the emotional tag of happiness that can be assigned to any stimulus that advances the goal of well-being. In a similar fashion numerous emotional tags can be given to stimuli that promote or hinder the attainment of goals ranging from basic individual survival goals to more complex social interaction goals. This vast range of emotions and the related goals is not likely to have been formed concurrently. Rather, emotions evolved from very simple mechanisms that ensured harm avoidance and attainment of vital physical resources into more complex mechanisms that guide complex social behavior. This evolution of emotions may in fact be reflected in the brain systems that generate them, with emotions linked to survival arising from evolutionarily old brain systems. Thus the more primitive emotions would be expected to be elicited by more primitive aspect of the environment, and only at higher levels of evolution would complex classification of stimuli have had related emotions associated with them [9].

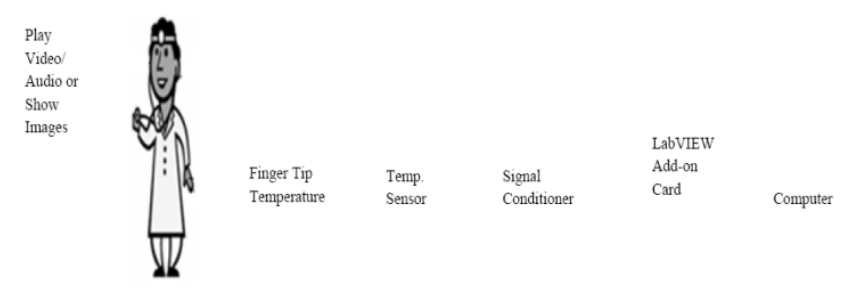

Fig. 1. Block diagram of the designed system

Fig. 1 illustrates the system for measuring Finger Tip Temperature. The subject under analysis is made to change the emotional status by external influence. Data are acquired 
in a closed laboratory condition, by playing the video/audio clippings or showing images. Table II shows the different movie clips used to evoke four prominent emotions i.e. happy, surprise, disgust and fear. Sufficient time is allowed in between so that the subject can reach the relaxed state which is considered as the neutral emotional status. Sufficient care is taken so that the subject's attention is not diverted from the intended task.

\section{EXPERIMENTS}

Many experiments have been conducted by researchers in search of universal physiological patterns, specific to basic emotions. Those studies concentrated mainly on activities of the autonomous nervous system (ANS) and characteristic speech signal changes. ANS related studies [1] showed very interesting results each on its own, but until now no distinct patterns for the six basic emotions could be found that all agree on. The results of the studies are controversial and the variables measured do not seem to allow clear distinction between different emotions. Some stable results could be found for variables that seem to characterize certain basic emotions, especially fear and anger, which are the two that previous literature have focused on mostly [2]. The work on ANS studies resulted in the following commonalities [3]. For fear: increase of heart rate, skin conductance level and systolic blood pressure; for anger: increase of heart rate, systolic and diastolic blood pressure. Measuring sadness seems to be more difficult. Because of this, the results are not as clear as for fear and anger. In paper [4], Erickson K. and Schulkin J. have found a decrease in heart rate, while paper [6] reported an increase of heart rate, while paper [7] could not find any significant difference to the measured baseline. Papers [5][10] and [11] relates emotion by and large to the facial expressions. Disgust seems to represent an even greater problem. It is assessed by four out of the 13 studies. The results are not very promising. Also, measuring positive emotions such as happiness seems to be very troublesome as can be observed especially in diastolic and systolic blood pressure and heart rate data [8], which can show a sign of decrease to even a strong increase.

The IC sensor corresponding to the finger tip temperature measurement is tied to the small finger. It is an accurate and hand held instrument which can be used without any inconvenience to the subject. Sensitivity control is provided to vary the response of the instrument to changes in FTT. Video clips are played for about six minutes and the values are recorded using the LabVIEW data acquisition add-on card as shown in Fig. 2.

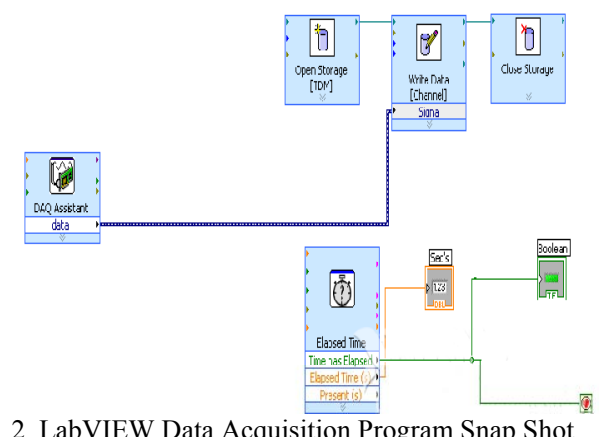

Fig. 2. LabVIEW Data Acquisition Program Snap Shot
TABLE I: INDEXING THE EMOTIONAL STATUS

\begin{tabular}{|l|c|c|c|c|c|}
\hline Emotion & Neutral & Fear & Disgust & Happy & Surprise \\
\hline Index & 1 & 2 & 3 & 4 & 5 \\
\hline
\end{tabular}

TABLE II: Theme OF MOVIE Clips CAUSING STIMUlATION OF SPECIFIC EMOTIONS

\begin{tabular}{|l|l|l|}
\hline Video source & $\begin{array}{l}\text { Emotion } \\
\text { stimulated by the } \\
\text { movie clip }\end{array}$ & $\begin{array}{l}\text { Theme of the movie } \\
\text { clip }\end{array}$ \\
\hline Kopfschlagen & Happy & Funny scene \\
\hline Funny Kids & Happy & Cute accidents of kids \\
\hline Amazing dance & Surprise & Dancing \\
\hline Atomic cannon & Surprise & Destruction \\
\hline $\begin{array}{l}\text { Snake eat } \\
\text { hippopotamus }\end{array}$ & Disgust & Snake eat hippopotamus \\
\hline Wrong turn & Fear & Cutting a human body \\
\hline See no evil & Fear & Plucking eyes \\
\hline Saw & Fear & Dissection of skull \\
\hline \hline
\end{tabular}

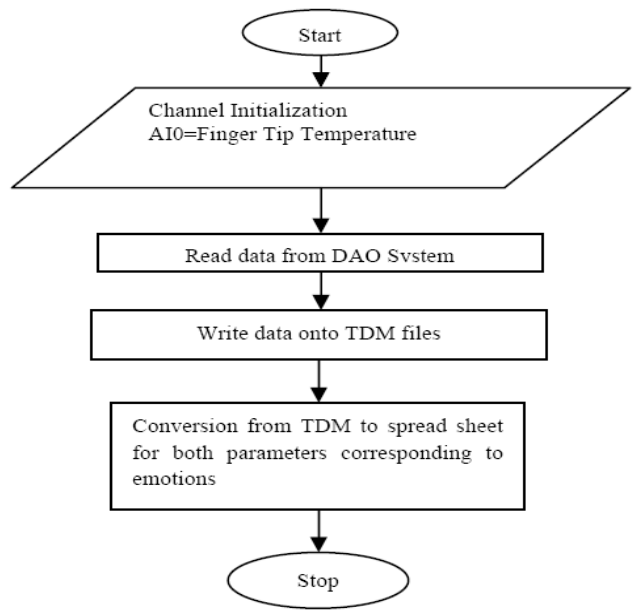

Fig. 3. Flow chart for acquisition of data and conversion of files

\section{IMPLEMENTATION}

The whole signal acquisition and analysis of emotions are carried out by using the graphical programming language LabVIEW. Flowchart shown in figure 3, depict the stages involved in physiological data acquisition and analysis respectively. The steps involved are as follows.

1) Connect the LabVIEW 48 channel analog and digital IO card to the USB port of the computer.

2) Configure channel0 as analog input channelsAI0.

3) Connect channel AI0 to finger tip temperature output.

4) Read data from the channel continuously after 120 seconds, while playing the stimulation input for 300 seconds. The read data will be automatically stored in a Technical Data Management (TDM).

5) Convert TDM file into spread sheet so that it can be stored as a database and accessed for further calculation and data processing.

6) Determine the maximum and minimum values and compute the average value.

7) Construct an array of average values for all subjects and store it in the spread sheet.

8) Connect the input of the sensor to the test subject, 
acquire data, find minimum \& maximum values and compute average value.

9) Compute the Manhattan or city block distance using equation (1), which is the equation for $\mathrm{L}_{\mathrm{p}}$ Norm.

$$
D\left(X, X^{\prime}\right)=\left(\sum_{k=1}^{d}\left|X_{k}-X_{k}^{\prime}\right|^{q}\right)^{\frac{1}{q}}
$$

where $\mathrm{q}=1$ and $\mathrm{d}=$ number of samples

10) Display one of the emotion index shown in Table I, for which Manhattan distance is minimum.

\section{RESUlts}

The changes in emotions under some stress are measured by considering the parameter: finger tip temperature. Finger tip temperature is obtained in terms of voltage and ${ }^{0} \mathrm{C}$ units. We are considering different emotion conditions such as neutral, fear, happy, disgust and surprise. Data is acquired and analyzed separately for both adult men and women from different age and background. A total of 75 subjects were considered for data collection. Out of the many stimulating video, the most effective shown in Table II are selected for final data collection. Data corresponding to FTT is shown in Table III. The duration of physiological signal acquisition of each emotion is 5-6 minutes. Graphs for the distribution of feature values for FTT corresponding to two different emotions are shown in Fig. 4(a) and 4(b).
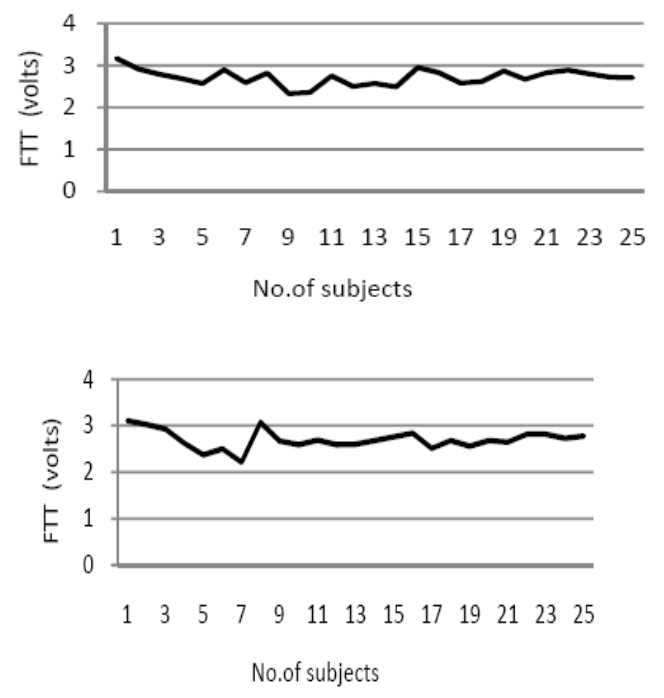

Fig. 4(a) Happ Fig. 4(b) Surprise

Fig.4. Plots for the distribution of feature values

TABLE III: PERCENTAGE ClASSIFICATION OF AROUSED EMOTIONS FOR SELECTED 10 SUBJECTS.

\begin{tabular}{|c|c|c|c|c|c|c|}
\hline Sl.No. & Subjects & Neutral & Fear & Disgust & Happy & Surprise \\
\hline 1. & Suma & 82.22 & 81.57 & 80.13 & 77.01 & 79.06 \\
\hline 2. & Vijaya & 76.92 & 75.18 & 70.54 & 98.58 & 88.76 \\
\hline 3. & Smitha & 80.82 & 75.72 & 82.34 & 72.13 & 88.96 \\
\hline 4. & Suvarna & 80.00 & 79.58 & 81.34 & 79.30 & 79.75 \\
\hline 5. & Kamala & 83.02 & 81.56 & 77.73 & 77.96 & 79.70 \\
\hline 6. & Manjula & 81.89 & 81.39 & 80.00 & 78.35 & 78.35 \\
\hline 7. & Sujatha & 83.46 & 82.39 & 80.01 & 76.38 & 77.73 \\
\hline 8. & Ashwini & 83.06 & 81.83 & 82.27 & 76.77 & 76.06 \\
\hline 9. & Geetha & 85.66 & 77.82 & 74.22 & 79.45 & 82.83 \\
\hline \multirow[t]{2}{*}{10.} & Jyothi & 81.73 & 80.17 & 91.16 & 91.66 & 92.09 \\
\hline & Average & 81.87 & 79.72 & 79.97 & 80.75 & 82.32 \\
\hline
\end{tabular}

\section{CONCLUSIONS}

The physiological status of a person is not always same: it changes according to time and the situations. We found that the result obtained by our system almost follows ground reality. However there is a small deviation which can be attributed to the subject under test and changing state of emotions due to external factors even though we tried to keep them at minimum. The new approach introduced in this paper provides a means for structuring, representing and processing emotions within a system without compromising the ambiguous nature of emotion. It does not claim to answer general questions on emotions, like what exactly are emotions or what makes them special in comparison to other mental states. We have collected a total of 750 data of physiological signal measurements from a diversified group of subjects like children, adults and senior citizens from both sexes, so that the study and analysis is almost comprehensive. By the study and analysis we can conclude that the percentage recognition is above $80 \%$ for most of the emotions. We can also conclude that compared to other emotions, it is difficult to stimulate and identify disgust. Also, it is very easy to evoke and identify positive emotions like happiness unlike negative emotions like fear and disgust. Use of more than one physiological parameter increases the robustness and reliability of the system.

\section{ACKNOWLEDGEMENTS}

The authors would like to thank the authorities of Malnad College of Engineering, Hassan, India for providing all facilities to do the research work at the institution and also the anonymous reviewers for the valuable comments and suggestions.

\section{REFERENCES}

[1] A. Achaibou, G. Pourtois, S. Schwartz, and P. Vuilleumier, "Simultaneous recording of EEG and facial muscle reactions during spontaneous emotional mimicry," Neuropsychological, vol. 46, no. 4, pp.1104-1113, 2008

[2] A. Castro, F. Diaz, and G. J. V. Boxtel, "What happens to the readiness potential when the movement is not executed," Neuroreport, vol. 16, no. 15, pp.1609-1613, 2005.

[3] A. R. Damasio, "Descartes' error: Emotion, reason and the human brain," Journal of Neurophysiology, vol. 2, pp. 29-40, 1994.

[4] K. Erickson and J. Schulkin, "Facial expressions of emotion: a cognitive neuroscience perspective," Brain and Cognition, vol. 52, no. 1 , pp. 52-60, 2003.

[5] U. Hess and R. E. Kleck, "Differentiating emotion elicited and deliberate emotional facial expressions," P. Ekman and E. L. Rosenberg (Eds), what the face reveals: Basic and applied studies of spontaneous expression using the facial action coding system (FACS) 2nd ed, pp. 271-286, Oxford University Press, 2005.

[6] P. K. Salmon, M. A. Henaff, A. Vighetto, F. Bauchet, O. Bertrand, and F. Mauguiere, "Experiencing and detecting happiness in humans: the role of the supplementary motor area," Annals of Neurology, vol. 59, no.1, pp.196-199, 2006.

[7] S. Korb, D. Grandjean and K. Scherer, "Investigating the production of emotional facial expressions: a combined electroencephalographic (EEG) and electromyographic (EMG) approach," IEEE International Conference on computational intelligence, pp.1-6, 2008.

[8] C. Peter and A. Herbon, "Emotion representation and physiology assignments in digital systems," interacting with Computers (Elsevier), no.18, pp.139-170, 2006.

[9] A. Ross and A. Jain, "Information fusion in biometrics", Pattern Recognition Letters (Elsevier), no. 24, pp. 2115-2125, 2003.

[10] G. J. Taylor and F. N. Fragopanagos, "The interaction of attention and emotion," Neural Networks (Elsevier), no.18, pp.353-369, 2005. 
[11] G. Shivakumar and P. A. Vijaya, "Face Recognition System Using Back Propagation Artificial Neural Network," International Journal of Computer Science and Information Technology, vol. 1, no. 1, pp. 68-77, 2009.

[12] G. Shivakumar, P. A. Vijaya, and R. S. Anand, "Artificial Neural Network Based Cumulative Scoring Pattern Method for ECG Analysis", IEEE International conference on Advances in Computer Vision and Information Technology, pp. 451-457, Aurangabad, 2007.

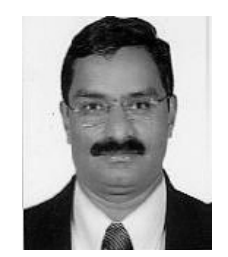

G. Shivakumar obtained his B.E. Degree in Instrumentation from University of Mysore, India and M.Tech. From Indian Institute of Technology, Kharagpur, India. Currently he is working as an assistant professor in the Department of Instrumentation at Malnad College of Engineering, Hassan, India. His fields of interest are biometrics, pattern recognition, image processing, artificial neural networks and embedded systems.

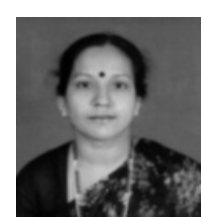

P. A. Vijaya received her M. E. and Ph.D. degree from the Department of CSA, I.I.Sc., Bangalore, India. She is currently working as Professor in the Department of E\&C Engineering of Malnad College of Engineering, Hassan, INDIA. She has around 50 research publications in international journals/conferences. She has been involved in the organization of a number of workshops and symposiums and other courses at MCE, Hassan. She is also a reviewer for a number of journals of National and International repute. Her main research interests are in pattern recognition, image processing, embedded systems, operating systems and computer architecture. 